\title{
TikTok as a data gathering space: the case of grandchildren and grandparents during the COVID-19 pandemic
}

\author{
Marije Nouwen \\ Meaningful Interactions Lab (Mintlab), KU Leuven \\ marije.nouwen@kuleuven.be
}

\begin{abstract}
Grandparents and grandchildren benefit from a relationship with each other. However, in times of crisis and social distancing it is challenging to maintain this relationship. In this paper, we reflect on TikTok as a data gathering platform that might help designers to capture and ideate on remote communication design across generations. We report on a study that aimed to learn about how grandparents and grandchildren perform their relationship on TikTok in 2020 during the COVID-19 pandemic. Our work might trigger discussions on the potentials of social media as a place for data gathering, as well as the implications of these insights when designing for and with children, and more particularly intergenerational remote interactions.
\end{abstract}

\section{CCS CONCEPTS}

\section{- Empirical studies in HCI; • Children; • Seniors;}

\section{KEYWORDS}

Tik Tok, COVID-19, Intergenerational Solidarity, social media, grandchildren, grandparents

ACM Reference Format:

Marije Nouwen and Mathilde Duflos. 2021. TikTok as a data gathering space: the case of grandchildren and grandparents during the COVID-19 pandemic. In Interaction Design and Children (IDC '21), June 24-30, 2021, Athens, Greece. ACM, New York, NY, USA, 5 pages. https://doi.org/10.1145/3459990.3465201

\section{INTRODUCTION}

The lives of children are affected severely during the COVID-19 pandemic, with limitations to socialization with peers and family members for instance. Households and generations are separated on a worldwide scale, due to barrier measures like keeping physical distance and self-isolation. Adults above 65 years old or with a pre-existing health condition run the potential risk of severe health complications, and even death, when they are infected with the virus. As a consequence, grandchildren have limited contact with their grandparents.

People of all ages, including grandchildren and their grandparents, use social media and digital technologies to keep in touch over distance. The widespread use of social media during the last decades

Permission to make digital or hard copies of part or all of this work for personal or classroom use is granted without fee provided that copies are not made or distributed for profit or commercial advantage and that copies bear this notice and the full citation on the first page. Copyrights for third-party components of this work must be honored

For all other uses, contact the owner/author(s).

IDC '21, fune 24-30, 2021, Athens, Greece

(c) 2021 Copyright held by the owner/author(s).

ACM ISBN 978-1-4503-8452-0/21/06.

https://doi.org/10.1145/3459990.3465201

\author{
Mathilde Duflos \\ Psychologie des Âges de la Vie et Adaptations (EA 2114, \\ PAVéA), University of Tours \\ mathilde.duflos1@gmail.com
}

has mediated and altered family relations and functioning [10]. Mobile social media were shown to enhance family solidarity, i.e., the positive and counter-productive aspects of belonging, among family members who live in separate households with various communication needs across different life-situations and within different family structures [14]. Intergenerational solidarity is mainly studied in face-to-face interactions, leaving a knowledge gap about how grandparents and grandchildren perform solidarity in the online environment.

In this paper, we look more specifically at TikTok, an online social media platform and mobile app that features videos up to 60 seconds, mostly for entertainment. The platform is characterized by a number of spontaneous videos, as well as content that needs to be rehearsed (like dance challenges) [17]. TikTok has 689 million active users worldwide [23]. As an emerging social network quickly adopted my many users, TikTok is gaining popularity among American teens (32,5\% of total users) and young adults (29,5\% of total users), as well as adults over 50 years old (7,1\% of total users). Therefore, studying this social network could be particularly relevant to understand various aspects of the lives of young people, with regards to their relationship with their grandparents. Recent media articles reported on TikTok as a platform for grandparents and grandchildren to stay connected by sharing dance videos with each other [22] or for including grandparents in youths' videos, for instance when dropping off groceries [11] during the COVID-19 pandemic. These examples shed light on the social media use of young people beyond their peer group to online interactions with their grandparents. We approach this phenomenon with the intergenerational solidarity framework, which is concerned with social cohesion and belonging across generations [7], by analyzing videos that grandchildren and grandparents posted during the COVID-19 pandemic in the year 2020. We present a study of 328 videos that focuses on the different forms of intergenerational solidarity [8] performed on the platform. Our contribution is on TikTok as a data gathering platform on the lives of young people, with a specific focus on their relationship with their grandparents, to suggest possibilities of TikTok as a data gathering platform for the IDC community.

\section{INTERGENERATIONAL SOLIDARITY}

The intergenerational solidarity framework proposes that different feelings and behaviors between grandparents and grandchildren define their relationship [12] and sense of belonging. The framework stipulates six dimensions of intergenerational solidarity, i.e., affectual solidarity, associational solidarity, consensual solidarity, functional solidarity, normative solidarity, structural solidarity [8]. 
Table 1: Six components of intergenerational solidarity framework

\begin{tabular}{|c|c|c|}
\hline Component & Definition & Examples \\
\hline Affectual solidarity & $\begin{array}{l}\text { Feelings of closeness between members of the same family and } \\
\text { their reciprocity }\end{array}$ & $\begin{array}{l}\text { Saying "I love you", hugging or } \\
\text { making jokes }\end{array}$ \\
\hline Associational solidarity & $\begin{array}{l}\text { Different types and patterns of contact and interactions between } \\
\text { members of the same family }\end{array}$ & $\begin{array}{l}\text { Visiting each other, dancing } \\
\text { together or sharing a meal }\end{array}$ \\
\hline Consensual solidarity & $\begin{array}{l}\text { Concordance of opinions, values, and beliefs between members of } \\
\text { the same family }\end{array}$ & $\begin{array}{l}\text { Sharing the same values and } \\
\text { beliefs, common political and } \\
\text { community commitments }\end{array}$ \\
\hline Functional solidarity & $\begin{array}{l}\text { Mutual help between generations, financial or non-financial, } \\
\text { instrumental or emotional }\end{array}$ & $\begin{array}{l}\text { Cooking, doing groceries, } \\
\text { expressing empathy or listening to } \\
\text { each other }\end{array}$ \\
\hline Normative solidarity & $\begin{array}{l}\text { Expectations toward filial and parental duties, and to the sense of } \\
\text { obligation related to a person's role within the family and } \\
\text { compliance with family norms and values }\end{array}$ & $\begin{array}{l}\text { Respecting filial piety and family } \\
\text { values }\end{array}$ \\
\hline Structural solidarity & $\begin{array}{l}\text { Opportunities for intergenerational contact facilitated by } \\
\text { geographical proximity }\end{array}$ & $\begin{array}{l}\text { When living in the same house or } \\
\text { when travelling to visit each other, } \\
\text { health status of family members }\end{array}$ \\
\hline
\end{tabular}

These six dimensions stem from interpersonal relationship theories [15]. The six components of intergenerational solidarity are presented in table 1 , below.

Our decision to focus on intergenerational solidarity emphasizes the role of young people as grandchildren on equal foot with their grandparents, which sheds light on children beyond their peer group. In the remainder of this paper, we focus on the behaviors and feelings of young people on TikTok during the COVID-19 pandemic in 2020 , by analyzing 328 videos that were posted by grandchildren or grandparents and that portray, in one way or another, their mutual relationship. The guiding research questions for this work are:

- How do grandparents and grandchildren perform intergenerational solidarity on TikTok during the COVID-19 pandemic?

- What are the potential uses of online research, i.e., on TikTok, to inform the design of technologies for grandchildren and grandparents?

We will first detail our method and then, share our preliminary results that highlight the potential of TikTok as a data gathering platform for the design of technologies for grandparents and grandchildren.

\section{METHOD}

The method followed a qualitative media analysis approach [2] of TikTok videos that include moving images, sound, written text, hashtags and the caption of the video. In January 2021, we created a new TikTok account for the purpose of the current research because TikTok algorithmically filters content based on user patterns [20]. This way, we avoided any prior personal search queries in the app to minimize any bias. Then, we followed a common practice in online research to create the data corpus by combining hashtags for data selection [16]. We combined relevant hashtags for our research questions in 6 search queries that all started with either grandchild or grandparent, followed by a synonym to the COVID19 pandemic (quarantine, lockdown or corona). For each search query, we selected up to 70 videos that were posted between March and December 2020. We made sure to only include videos in the languages we master (i.e., English, Dutch, French, Spanish, German) and not to include videos that were selected for a previous search query. Duplicates were frequent, which had as a consequence that we reached the limit of the TikTok feed before arriving at the threshold of 70 videos for some search queries. An overview of the search queries with the number of selected videos is in table 2

We compiled all the data in an Excel sheet and saved the selected videos on the first author's university data storage network. The data sheet is divided into three large categories including information of the video, transcription and coding. Table 3 stipulates the different data types that we included for analysis. We downloaded most of the videos from the TikTok mobile app or proceeded with iPhone's screen recorder when this was not possible. We did not inform individual users as this would entail a disproportional distribution of information for publicly shared video content. We took measures to protect the privacy of participants by excluding usernames or other personal identifiable information in our Excel sheet, and deleting all videos from personal devices after analysis. The research protocol received approval from the social and societal ethics committee at the faculty of the first author.

After compiling all the data in an Excel sheet, we transcribed the videos (see table 3 , middle column). Then, we proceeded with the first coding round following the six dimensions of the intergenerational solidarity framework in an adaptive theory approach [19] (see table 3, third column). We made sure to check for intercoder reliability to improve transparency and reflexivity among both authors [21] by individually coding letter code B. This resulted in 33 matches and 37 videos that received a different code from one of the two coders. The results fell short of our expectations, which led to thorough discussions to obtain a shared understanding of the different dimensions of our theoretical coding book. Videos that 
Table 2: Six search queries with corresponding letter code and number of selected videos

\begin{tabular}{lll}
\hline Letter code & Search query & Number of selected videos \\
A & \#grandparent \#quarantine & 69 \\
B & \#grandparent \#lockdown & 70 \\
C & \#grandparent \#corona & 60 \\
D & \#grandchild \#quarantine & 35 \\
E & \#grandchild \#lockdown & 70 \\
F & \#grandchild \#corona & 24 \\
\hline
\end{tabular}

Table 3: Components of the data sheet

\begin{tabular}{lll}
\hline Information of the video & Transcription & Coding \\
Number & Music (sound title) & Affectual \\
Search query letter code & Spoken words & Associational \\
Search date & Written text (on moving image) & Consensual \\
Upload date & Filter & Functional \\
Number of comments & People and animals & Normative \\
Number of likes & Ethnicity & Structural \\
Amount of shares & Actions & \\
Hashtags & Background & \\
Caption & Link with other video & \\
Duration (\# seconds) & Development stage of the grandchild & \\
URL & Person who shares the video & \\
\hline
\end{tabular}

led to disagreements were often at the border between two dimensions of intergenerational solidarity (e.g., visiting a grandparent or grandchild with an act of affection, functional support with a cheering card or verbalizing affection for the other). These discussions resulted in a set of concrete guidelines for further coding steps. Then, author 2 continued coding the videos with letter code $A$ and $\mathrm{C}$, and author 1 coded the videos with letter code $\mathrm{D}, \mathrm{E}$ and $\mathrm{F}$. This is the current stage of our analysis process. We plan to continue our analysis to identify subthemes and look for patterns in and across the different coding dimensions. In the remainder of this paper, we share our preliminary results and highlight the potential of TikTok as a data gathering platform for the IDC community.

\section{PRELIMINARY RESULTS}

The qualitative media analysis of 328 TikTok videos, posted during the COVID-19 pandemic in 2020, sheds light on the lives of grandchildren of all ages; 75 videos feature newborns, 22 videos include toddlers, 33 children feature children (including two videos of babies and children), 115 videos include adolescents (including four videos with adolescents and children) and 81 include (emerging) adults (and three videos where the age could not be identified). 57\% of the videos was shared by grandchildren, $22 \%$ by grandparents, $17 \%$ by parents of grandchildren and $4 \%$ by other parties. We discuss the results through the six dimensions of the intergenerational solidarity framework.

Videos coded under affectual solidarity are most present in our data $(n=127)$ and highlight interactions through affective verbal communication and gestures. Grandchildren were found to be creative in their ways to cuddle their grandparents while respecting physical barriers (e.g., by wearing full body suits or hugging with a plastic wall in between two bodies). Hugging without these measures was observed when regulations became less strict. Grandchildren posted recent or old pictures to express their affection towards their grandparents when physical contact was not possible. Baby grandchildren, in turn, were often present in picture compilations even when it had not been possible to visit the baby themselves. Celebrations often included physical-distanced parties or gestures (e.g., when inviting a mariachi band to play outside a grandmother's window). Some videos found ways to laugh with the restrictions (e.g., grandparents act like spies while delivering supplies to a grandchild).

Associational solidarity is the second most present dimension in our data set $(n=91)$. Many videos show different ways that grandparents and grandchildren keep entertained by playing with baby or child grandchildren (including rotating a desk chair and shooting Nerf guns). Other videos show how grandparents and grandchildren participate in TikTok challenges together, mostly with 'Laxed (Siren Beat)' by Jawsh 685 or 'Something New' by Wiz Khalifa' feat. Ty Dolla \$ign. These challenges mainly include teenaged grandchildren and their grandparents, or different ages or grandparents without grandchildren. Other examples include baking together or doing each other's hair and make-up.

Consensual solidarity is the least present in our data set $(n=3)$, which implies that grandchildren and grandparents express opinions, values and beliefs to a lesser extend during the COVID-19 pandemic on TikTok. Our data includes a video of an emerging adult grandson who films his reactions to his grandparents' acceptance of his outing as a gay man, a granddaughter who cuts her grandfather's political party membership card in two, and a 
granddaughter who tricks her grandmother into dancing to a song that glorifies smoking marihuana.

Functional solidarity was present in 49 videos in our data set. Most videos include grandchildren or grandparents dropping off or picking up (homemade) food or non-essential items like flowers or jackets. In other videos we see grandchildren doing house chores, like groceries, painting sheds or gardening. Grandchildren also offered physical support (e.g., by sharing stretching exercises via a tablet), technical support (e.g., sharing highlights of moments were the grandparents needed help during a family video call) and emotional support.

Normative solidarity was present in 38 videos in our data set. Most videos were themed around meeting baby grandchildren for the first time. Other videos included pregnancy announcements to the soon-to-be grandparents or great-grandparents. Normative solidarity was also reflected by grandchildren stating that they were the favorite grandchild when they received special treats (e.g., a teenage grandchild is invited to a nail treatment by her grandmother) or when grandchildren displayed how they protected their grandparents from getting infected (e.g., a grandchild runs after her grandfather to check if he is carrying his face mask and hand sanitizer before driving off).

Structural solidarity was present in 20 videos of our data set. This dimension was expressed through emphasizing the health of a grandparent and how families adapt their interactions (e.g., an adult granddaughter whose grandmother had a stroke and is in a wheelchair). Other videos emphasized the long distance they had to overcome to visit each other or, in contrast, how grandchildren moved in with their grandparents to keep them company during lockdown.

Overall, our results suggest that TikTok, like other social media, shapes the lives of grandchildren and grandparents alike. During an exceptional time, due to a global pandemic, mainly teenaged grandchildren and the parents of children expressed the importance of the grandparent-grandchild relationship and posted about them on TikTok, most evidently when bringing groceries, staying over to keep them company or finding ways to hug each other in a safe way. In the same vein, grandparents experienced the importance of having grandchildren and shared videos on this topic on TikTok, for instance when becoming grandparents. This implies that TikTok provides snapshots into the lives of people of all ages, even when the bulk of the user group is pre-adult. Moreover, the scope of our data set was beyond our imagination in terms of the variety of videos and backgrounds we encountered, despite our limited knowledge of world languages.

\section{REFLECTIONS ON TIK TOK FOR INTERACTION DESIGN}

This paper reports on a study that aimed to learn about how grandchildren and grandparents perform their relationship on TikTok in 2020 during the COVID-19 pandemic. Moreover, our goal was to explore the potentials of TikTok as a place for data gathering practices.

The results of this study give insight into the different ways that grandparents and grandchildren perform intergenerational solidarity on TikTok. Reflections based on the first round of analysis hint towards promising insights to contradictory behaviors during a global health crisis. Where some grandchildren made sure their grandparents were taking enough measures to protect themselves from the virus (under normative solidarity) others moved in with their grandparents to avoid isolation (under structural solidarity). Moreover, we expected to learn about the lives of teenaged grandchildren, as they represent the largest user group of the platform. However, we also learned about babies, toddlers, young children, adults and elderly people. It transposes the phenomenon of sharenting from parents with young or disabled children [9] across family members, including teenaged grandchildren and grandparents. Although sharenting is often motivated by feelings of pride, privacy issues remain [4]. Older adults might have insufficient knowledge of privacy and security management on an individual level [18] on online platforms that blur the boundaries between the private and public. Therefore, we will explore the indirect use by both (young) grandchildren and grandparents [6] and what the implications of these sharing practices are for the sense of belonging to the same family. We are confident our insights are relevant for others that, for instance, support children's use of social media to sustain relationships when physical contact is hindered [3] or provide solutions to child online safety [5].

Recent work in Human-Computer Interaction highlighted the potential of social media, like Instagram, for collecting mundane snapshots in design practice [1]. Our work reinforces the potential of social media to gather unexpected real-life snapshots for research and design purposes. The videos in our data set present a collage of different family practices, cultures and feelings regarding the grandparent-grandchild relationship. Many of the videos were spontaneous, showing clutter, messy kitchens and people in pyjamas. The "messines" of the video content stands in contrast with other research methods like interviews, surveys or experiments that are prone to practices of social desirability when participants want to perform well [13]. In this way, TikTok might provide insight in early stages of design to explore different contexts, without the presence of an external observer. Additionally, physical data gathering was complicated during the pandemic, which makes TikTok particularly convenient to study families online, even when social media are still relatively unexplored as a data gathering environment.

\section{ACKNOWLEDGMENTS}

The first author receives funding in the context of the re:tangent research project, funded by FWO (Fonds Wetenschappelijk Onderzoek Vlaanderen - grant number G0E3718N) in collaboration with the Center for Human-Computer Interaction (University of Salzburg).

\section{REFERENCES}

[1] Ferran Altarriba Bertran, Laia Turmo Vidal, Ella Dagan, Jared Duval, Elena Márquez Segura, and Katherine Isbister. 2020. Chasing Play with Instagram: How Can We Capture Mundane Play Potentials to Inspire Interaction Design? In Extended Abstracts of the 2020 CHI Conference on Human Factors in Computing Systems (CHI EA '20), 1-8. https://doi.org/10.1145/3334480.3382913

[2] David L. Altheide and Christopher J. Schneider. 2013. Qualitative Media Analysis. SAGE Publications, Ltd, London. https://doi.org/10.4135/9781452270043

[3] Alissa N. Antle and Christopher Frauenberger. 2020. Child-Computer Interaction in times of a pandemic. International fournal of Child-Computer Interaction 26: 100201. https://doi.org/10.1016/j.ijcci.2020.100201 
[4] Ulla Autenrieth. 2018. Family photography in a networked age: Anti-sharenting as a reaction to risk assessment and behaviour adaption. In Digital Parenting. The Challenges for Families in the Digital Age (Yearbook 2018), Giovanna Mascheroni, Cristina Ponte and Ana Jorge (eds.). NORDICOM, University of Gothenburg, 219-231. Retrieved from https://research.unl.pt/ws/portalfiles/portal/12488016/ yearbook_2018_digital_parenting.pdf\#page $=221$

[5] Karla Badillo-Urquiola, Diva Smriti, Brenna McNally, Evan Golub, Elizabeth Bonsignore, and Pamela J. Wisniewski. 2019. Stranger Danger! Social Media App Features Co-designed with Children to Keep Them Safe Online. In Proceedings of the 18th ACM International Conference on Interaction Design and Children (IDC '19), 394-406. https://doi.org/10.1145/3311927.3323133

[6] Eric P. S. Baumer and Jed R. Brubaker. 2017. Post-userism. In Proceedings of the 2017 CHI Conference on Human Factors in Computing Systems (CHI '17), 6291-6303. https://doi.org/10.1145/3025453.3025740

[7] V. L. Bengston and P. S. Oyama. 2007. Intergenerational Solidarity: Strengthening Economic and Social Ties. New York.

[8] Vern L. Bengtson and Robert E. L. Roberts. 1991. Intergenerational Solidarity in Aging Families: An Example of Formal Theory Construction. Fournal of Marriage and Family 53, 4: 856-870. https://doi.org/10.2307/352993

[9] Katya Borgos-Rodriguez, Kathryn E. Ringland, and Anne Marie Piper. 2019. MyAutsomeFamilyLife: Analyzing Parents of Children with Developmental Disabilities on YouTube. Proceedings of the ACM on Human-Computer Interaction 3 , CSCW: 94:1-94:26. https://doi.org/10.1145/3359196

[10] Joana Carvalho, Rita Francisco, and Ana P. Relvas. 2015. Family functioning and information and communication technologies: How do they relate? A literature review. Computers in Human Behavior 45: 99-108. https://doi.org/10.1016/j.chb. 2014.11.037

[11] Lindsay Dodgson. TikTokers are dancing with their grandparents for content and it could help curb the pandemic of loneliness among older people. Insider. Retrieved April 2, 2021 from https://www.insider.com/how-tiktok-bringsgrandparents-and-grandchildren-together-2020-6

[12] Mathilde Duflos and Caroline Giraudeau. in press. Using the intergenerational solidarity framework to understand the grandparent-grandchild relationship: a scoping review. European fournal of Ageing. https://doi.org/10.1007/s10433-02100626-6
[13] Pieter Duysburgh and Karin Slegers. 2015. Reciprocity in Rapid Ethnography. In Human-Computer Interaction - INTERACT 2015 (Lecture Notes in Computer Science), 292-299. https://doi.org/10.1007/978-3-319-22668-2_23

[14] Riitta Hänninen, Sakari Taipale, and Armi Korhonen. 2018. Refamilisation in the broadband society - the effects of ICTs on family solidarity in Finland. Fournal of Family Studies 0, 0: 1-17. https://doi.org/10.1080/13229400.2018.1515101

[15] Fritz Heider. 1958. The psychology of interpersonal relations. Wiley, New York.

[16] Sarah J. Jackson and Brooke Foucault Welles. 2015. Hijacking \#MYNYPD: Social Media Dissent and Networked Counterpublics. Journal of Communication 65, 6: 932-952. https://doi.org/10.1111/jcom.12185

[17] Daniel Klug. 2020. "It took me almost 30 minutes to practice this". Performance and Production Practices in Dance Challenge Videos on TikTok. arXiv:2008.13040 [cs]. Retrieved March 1, 2021 from http://arxiv.org/abs/2008.13040

[18] Jess Kropczynski, Zaina Aljallad, Nathan Jeffrey Elrod, Heather Lipford, and Pamela J. Wisniewski. 2021. Towards Building Community Collective Efficacy for Managing Digital Privacy and Security within Older Adult Communities. Proceedings of the ACM on Human-Computer Interaction 4, CSCW3: 255:1-255:27. https://doi.org/10.1145/3432954

[19] Derek Layder. 1998. Sociological practice. Linking theory and social research. Sage, London.

[20] Bernard Marr. AI In China: How Buzzfeed Rival ByteDance Uses Machine Learning To Revolutionize The News. Forbes. Retrieved April 11, 2021 from https:/www.forbes.com/sites/bernardmarr/2018/12/05/ai-in-china-howbuzzfeed-rival-bytedance-uses-machine-learning-to-revolutionize-the-news/

[21] Cliodhna O'Connor and Helene Joffe. 2020. Intercoder Reliability in Qualitative Research: Debates and Practical Guidelines. International fournal of Qualitative Methods 19: 1609406919899220. https://doi.org/10.1177/1609406919899220

[22] Sydney Page. Grandparents are dancing with their grandkids on TikTok. People can't get enough. Washington Post. Retrieved April 2, 2021 from https://www.washingtonpost.com/lifestyle/2020/05/14/grandparents-aredancing-with-their-grandkids-tiktok-people-cant-get-enough/

[23] Most used social media 2021. Statista. Retrieved April 11, 2021 from http://www.statista.com/statistics/272014/global-social-networks-rankedby-number-of-users/ 